\title{
Giant Maculo-Papular Speckled Lentiginous Nevus Associated with Isolated and Centrofacial Located Lentiginosus
}

\section{Betul Tas ${ }^{1 *}$ and Mehmet Sar ${ }^{2}$}

${ }^{1}$ Bagcilar Research and Training Hospital, Department of Dermatology, Turkey

${ }^{2}$ Bakirkoy Dr. Sadi Konuk Research and Training Hospital, Department of Pathology, Istanbul, Turkey

\begin{abstract}
Lentigines are usually flat, pigmented macules on the skin and mucosa. These lesions are characterized by mostly smaller than $0.5 \mathrm{~cm}$, irregular borders and different shades of brown and black. They can be in the form of circumscribed or widespread lesions, and occur either as isolated lesion or as a part of some congenital syndromes. The speckled lentiginous nevus is also named as Nevus Spilus which is a peculiar acquired lentiginous nevus, which can be divided into two distinct clinicopathological variants as macular and papular. On the other hand, centrofacial lentiginosus is a special form of congenital lentiginosus which is inherited as autosomal dominant, and is usually associated with some neurological and psychiatric findings. We report a 43-year old woman with a giant maculo-papular speckled lentiginous nevus associated with a different and non-syndromal isolated centrofacial lentiginosus. To the best of our knowledge this association has not been reported previously.
\end{abstract}

Keywords: Speckled lentiginosus nevus; Nevus spilus; Giant; Isolated; Centrofacial lentiginosus

\section{Introduction}

Nevus Spilus (NS) is a special lentiginous nevus which was divided into two different clinicopathological types by Happle. Macular form is characterized by flat and dark brown spots on a light-brown background, while papular form is characterized by dark brown papules or nodules on a light-brown background, although small dark spots may also be present [1]. On the other hand, the term "speckled lentiginous nevus (SLN)" was first introduced to literature by Stewart el al.in 1978 [2,3]. Today, the terms NS and SLN are used interchangeably, although first one is usually used to describe smaller lesions while second is usually preferred to describe extensive lesions [3]. On the other hand, centrofacial lentiginosis (CFL) was first described by Touraine as considered to be a consequence of skull-base dysraphia and to be related to nerve damage, psychiatric symptoms and pigmentary disorders. This lesion is usually inherited as an autosomal dominant. However, rarely, the lesion can be seen as an isolated phenomenon [4].

\section{Case}

A 43-year-old Caucasian woman was admitted to outpatient clinic of our dermatology department seeking a cosmetic remedy for her extensive skin pigmentations. According to the personal history, when the patient was 2-years old, her mother noticed some pigmented spots on the face and these lesions became more prominent especially in the middle of the face in the course of following ten years. Thereafter, she noticed similar pigmented spots on the left side of the trunk and left arm, when she was 8 years old. Initially, all pigmentations were indistict, but they gradually darkened and increased in number with the advancement of her age. In her family history, similar face and trunk lesions were found in her mother. Additionally, her mother died from breast cancer ten years ago. The patient has Fitzpatrick-IV skin type. In the dermatological examination, extensive unilateral and speckled pigmented plaques which were approximately $35 \times 40 \mathrm{~cm}$ in sizes located between T3 and L3 dermatoms on both front and back surface of the left side of the trunk, and similar speckled pigmented lesions which were located on inner-lateral surfaces of ipsilateral left arm and forearm were seen. The trunk lesions were composed of light-brown and brown macules, and a small number of papules on a very lightbrown background pigmentation. They did not exceed the midlines of the trunk, and especially front-trunk lesions sharply ended at the linea alba (Figure 1a and 1b), and complied with Type-II cutaneous mosaicism pattern (Figure $2 \mathrm{a}$ and $2 \mathrm{~b}$ ). Face spots accumulated especially on the nose, cheeks and chin, but some scattered spots were detected in peripheral regions of the face and also on the vermillion border of the lips (Figure 1c). There were no additional pigmented or other skin lesions on the rest of the skin and mucosal surfaces. On dermatoscopic examination of the trunk lesions, there was a thin and very-light brown colored pigment network on the background of maculopapular lesions, except for some periareolar regions. In order to determine the depth of the lesions, a Wood light examination was

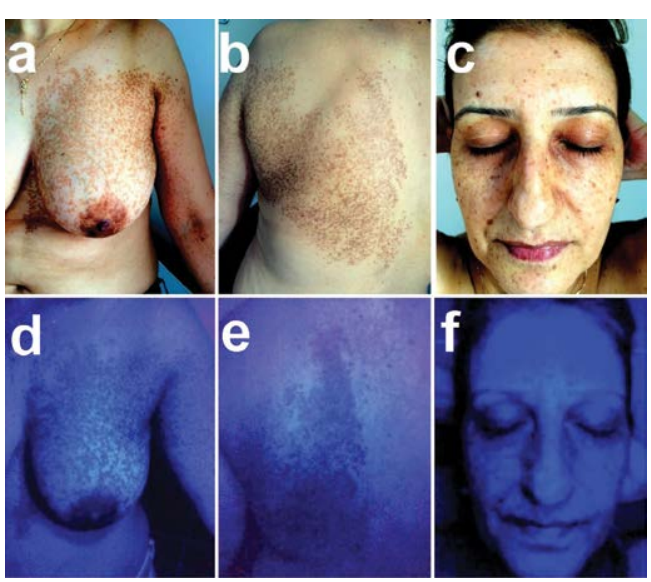

Figure 1: The clinical views of the trunk and face lesions $(a, b, c)$, and their appearances under the Wood light (d,e,f).

*Corresponding author: Betul Tas, Department of dermatology, Bagcilar Research and Training Hospital, Atakoy 7-8. Kisim, Marti Sitesi, 14/105, Bakirkoy Istanbul, 34156, Turkey, Tel: (+90) 212-4404000; Fax: (+90) 212-4404000; E-mail: betulavc@yahoo.com

Received April 06, 2015; Accepted June 03, 2015; Published June 15, 2015

Citation: Tas B, Sar M (2015) Giant Maculo-Papular Speckled Lentiginous Nevus Associated with Isolated and Centrofacial Located Lentiginosus. Pigmentary Disorders 2: 195. doi:10.4172/2376-0427.1000195

Copyright: () 2015 Tas B, et al. This is an open-access article distributed under the terms of the Creative Commons Attribution License, which permits unrestricted use, distribution, and reproduction in any medium, provided the original author and source are credited. 


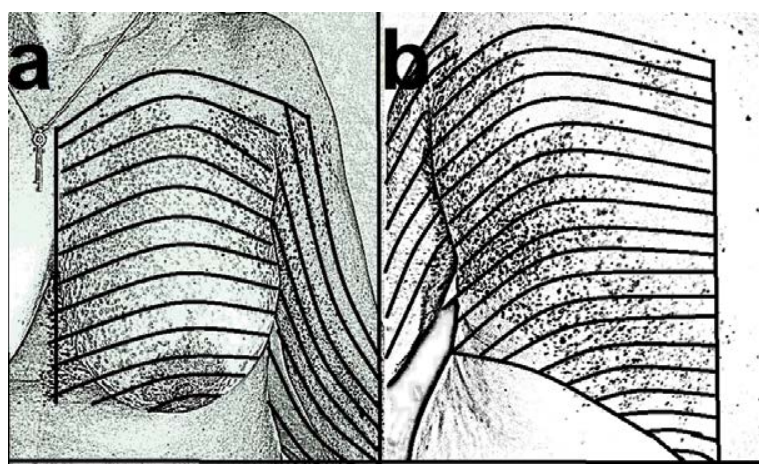

Figure 2: The flag-like configuration of the trunk lesions complied with the type-II mosaicism pattern $(a, b)$.

performed, and found that macular trunk lesions were faded while the background pigmentation and papular lesions were more prominent. All face lesions were faded under the Wood light (Figure 1d,1e and 1f). In the excisional skin biopsy of the trunk lesions, slight epidermal thickening, irregularly-arranged, moderately-pigmented, hyperplastic but isomorphic melanocytes in the basal keratinocytes, moderatelypigmented and increased clusters of similar-sized melanocytic nests along dermoepidermal junction, as well as more intense clusters of melanocytic nests at the top of the dermal papillae, and scattered ones in different levels of the papillary dermis were seen. Because the patient did not give consent to take a skin biopsy, we could not perform a histopathological examination from the face lesions. In the neurological examination, no findings supporting neurological pathologies were detected, and the psychiatric examinations of the patient were normal. The rest of the systemic examinations including breast, genito-urinary, gastrointestinal and cardiovascular systems, and ophtalmological examination were evaluated as normal. There was no regional or systemic lymphadenomegaly, or hepatosplenomegaly. The routine laboratory examinations, including blood cell count, blood biochemistry, blood sedimentation rate and urinalysis, as well as serum LDH levels, cancer markers were within the normal levels or negative. Performed chest radiogram, mammography and taken cervical smear, did not show any pathology. With these findings, the trunk lesions of the patient were diagnosed as maculo-papular speckled lentiginous nevus, and face lesions as centrofacial lentiginosis. No therapy was performed to the patient for the lesions. After the patient was informed about her pigmentations, she was advised to undergo routine dermatological check-ups especially if she notices any changes in the pigmented lesions or de-nova pigmented lesion.

\section{Discussion}

The prevalence of SLN is about $1-2 \%$ [3]. SLN reflects Type-II pattern (chequerboard-pattern) of cutaneous mosaicism, which is characterized by flag-like areas with limited a sharp body-midline $[2,5]$. The lesions can be sporadic or familial. Our case was a familial type. Familial cases show a paradominant trait $[2,3]$. The macular form can be isolated or a component of phacomatosis pigmentovascularis type-III, but it usually has a stable course. It can be seen at birth, or begins in the first few years of life [3]. Papular form can also be isolated, or a component of phacomatosis pigmentokeratotica (phacomatosis spilorosea) or SLN syndrome (papular-type SLN, ipsilateral hyperhidrosis, dysesthesia and muscle weakness) $[1,3,6]$. Macular forms may show a malignant degeneration. In the papular form, it is thought that the malignant degeneration is lower than that of macular type, but this form may be progressive [3,5]. Melanoma arising from a NS is a rare condition. It has been suggested that the reason for the difference of malignant potential between the two types of SLN, could be related to differences in the origins of mutations. Stella et al. have reported a nodular-melanomagenesis arising from a preexisting giant NS maculosus. In the macular form, the background pigmentation and brown spots correspond to hyperpigmention of basal keratinocytes, and junctional melanocytes arranged in nests, respectively. In the histopathology of papular forms, the features of superficial and deep congenital nevus in which nests of melanocytes are seen in the papillary and reticular dermis [1]. The clinical and histopathological findings of the trunk lesions of our patient complied with both macular and papular forms of SLN. Because our patient did not have any syndromal symptoms, her trunk lesions were considered to be non-syndromal form of SLN. Additionally, due to the fact that especially macular forms of SLN can be associated with malignancies, and the mother of our patient died from breast cancer, we examined our patient against the possibility of any malignancy, but it was unable to be determined. In differential diagnoses, primarily, partial unilateral lentiginosis (PUL) which seems to be a mosaic manifestation of type-1 neurofibromatosis, should be considered. Although histopathological findings of lentigines occuring in PUL and SLN are identical to each other, PUL can be differentiated from SLN with clinical features because the background pigmentation is absent in the former [1,7]. Rarely, in some areas of some SLN cases, the background pigmentation can also be absent, as seen in some periareolar regions of our patient. Therefore, some authors have suggested that SLN and some lentiginous conditions could be parts of the same spectrum [2]. Also, the diagnosis of phacomatosis spilorosea was excluded because of the absence of telangiectasias [1]. On the other hand, CFL was first described by Touraine in 1941. The characteristic feature of the lentigines in CFL is the presence of numerous lesions on the nose and cheeks in a "butterfly-like" pattern. They sometimes also occur on the forehead, eyelids and lips, and usually begin to occur in the first year of life, continue to persist up to the age of 8-10 years, and thereafter they tend to regress. Mucous membranes are spared. Although all reported cases by Touraine were younger than 40 years old, Dociu et al. described some patients who were older than 54 . CFL is usually inherited as autosomal dominant. Apart from the lentigines, CFL is associated with some neurological-dysraphic anomalies and psychiatric features. The dysraphic findings are olympian forehead, pectus carinatum, absence of xiphoid process, umblical hernia, cervico-dorsal kyphosis, spina bifida, foveola coccygea and lumbar hypertrichosis, while neuropsychiatric ones are oligophrenia, emotional instability, disturbance of behavior/ character, and, epilepsy, infantil hemiplegia and EEG abnormalities in some cases [4]. The other lentiginous conditions which are located on the middle of the face are Carney complex (CC), Peutz-Jeghers Syndrome (PJS) and inherited patterned lentiginosis in black persons (IPLBP). CC is also known as LAMB or NAME syndrome, comprises centrofacial lentigines which involve conjunctiva and the vermilion border of the lips, cardiocutaneous myxomas, blue nevi and some endocrine abnormalities. PJS is another autosomal dominant inherited lentiginous syndrome consisting of pigmented spots on the lips, oral mucosa, perioral areas and dorsal parts of acral areas as well as polyps of gastrointestinal, respiratory and renal systems. With time, lip lentigines may fade, but mucosal ones do not. IPLBP is seen in black patients whose lentigines are distributed over the central part of face and lips, with variable involvement of the dorsum of the hands and feet, elbow, and buttocks, and inherited autosomal dominant. The mucosae are spared [8]. Histopathologically, all the lentigines show similar findings $[8,9]$. Since our patient was not black, her facial lentigines were located on the face and lips but not in mucosa, and absence of any syndromal relation, they were not thought to be associated with the mentioned 
Citation: Tas B, Sar M (2015) Giant Maculo-Papular Speckled Lentiginous Nevus Associated with Isolated and Centrofacial Located Lentiginosus. Pigmentary Disorders 2: 195. doi:10.4172/2376-0427.1000195

Page 3 of 3

lentiginous conditions. Due to the history of presence of similar lesions in mother of the patient, we thought the both trunk and face lesions were familial. Additionally, because the facial spots were dissimilar to previously defined centrofacial lentigines, they were thought to be a different and isolated variant of centrofacial-located lentiginosus. Our patient was presented, because the coexistence of combined type of SLN and isolated and non-syndromal centrofacial-located lentiginosus have not been reported previously.

\section{References}

1. Stella A, Ponholzer K, Weingast J, Binder M (2011) Melanoma arising in a gian nevus spilus maculosus. Dermatol Pract Concept 1: 21-24.

2. Crosti C, Betti R (1994) Inherited extensive speckled lentiginous nevus with ichthyosis: report of a previously undescribed association. Arch Dermatol 130: 393-395.

3. Vidaurri-de la Cruz H, Happle R (2006) Two distinct types of speckled lentiginous nevi characterized by macular versus papular speckles. Dermatology 212: 53 58.

4. Dociu I, Galaction-Niţelea O, Sirjiţã N, Murgu V (1976) Centrofacial lentiginosis A survey of 40 cases. Br J Dermatol 94: 39-43.

5. Happle R (2002) Dohi Memorial Lecture. New aspects of cutaneous mosaicism. J Dermatol 29: 681-692.

6. Vente C, Neumann C, Bertsch H, Rupprecht R, Happle R (2004) Speckled lentiginous nevus syndrome: report of a further case. Dermatology 209: 228229.

7. Happle R (2007) Nevus spilus maculosus vs. partial unilateral lentiginosis. J Eur Acad Dermatol Venereol 21: 713.

8. Stratakis CA (2000) Genetics of Carney complex and related familial lentiginoses, and other multiple tumor syndromes. Front Biosci 5: D353-366.

9. Lodish MB, Stratakis CA (2011) The differential diagnosis of familial lentiginosis syndromes. Fam Cancer 10: 481-490. 